\title{
Análise Empírica de Métodos de Localização de Terminais GSM Legados utilizando Predição de Cobertura e Alinhamento Temporal
}

\author{
Rafael Saraiva Campos e Lisandro Lovisolo
}

\begin{abstract}
Resumo-Neste trabalho são analisados quatro métodos de localização de terminais móveis GSM legados. Estes métodos produzem uma estimativa de posição através da correlação de parâmetros disponíveis no relatório de medidas enviado pelo móvel com assinaturas de rádio-freqüência. As assinaturas de rádio-freqüência empregadas são preditas por modelagem de rádio-propagação. Este trabalho propõe uma alternativa para a utilização do alinhamento temporal do terminal móvel com a célula servidora para redução do erro de localização. Testes de campo avaliaram o desempenho dos diferentes métodos em cenários reais.
\end{abstract}

Palavras-Chave-Localização de Estação Móvel, Assinatura de Rádio-Freqüência, Predição de Cobertura, Alinhamento Temporal.

Abstract-In this work four methods for legacy GSM mobile station (MS) positioning are analyzed. These methods produce an estimative of the mobile station position by correlating the parameters reported by the MS in the measurement report with radio-frequency signatures. The employed radio-frequency signatures are obtained by propagation modeling. An alternative use of the time alignment between the MS and the serving cell for reducing the positioning error is presented. Field tests were conducted to evaluate the performance of the methods in real world scenarios.

Keywords-Mobile Station Positioning, Radio-Frequency Signature, Coverage Prediction, Time Alignment.

\section{INTRODUÇÃO}

Em vários países, órgãos reguladores têm especificado requisitos para a localização de telefones móveis celulares (MS - mobile stations) [1], [2] originando chamadas para números de emergência. O fórum responsável pelas normas do padrão GSM (Global System for Mobile Telecommunications) definiu novos elementos de rede e interfaces a serem incorporadas a redes GSM para suportar a localização precisa do MS [3]. No Brasil, a ANATEL (Agência Nacional de Telecomunicações) ainda não adotou normas semelhantes, contudo isto pode tornar-se realidade num futuro próximo. Neste cenário, as operadoras deverão ser capazes de estimar a posição de qualquer MS originando uma chamada de emergência. Aproximadamente $80 \%$ dos celulares em uso no país são GSM e cerca de $81 \%$ dos usuários utilizam serviço pré-pago [4], com baixo consumo médio mensal e modelos simples de aparelhos. Estes dados indicam que a maioria absoluta dos telefones móveis celulares no Brasil é composta de terminais legados - i.e., sem qualquer tipo de suporte específico a serviços de localização (LCS - Location Services). Assim, torna-se essencial a proposição de alternativas para a localização dos MS GSM legados. Além disso, esses métodos

Rafael Saraiva Campos e Lisandro Lovisolo, Faculdade de Engenharia, Programa de Engenharia Eletrônica, Universidade Estadual do Rio de Janeiro, Rio de Janeiro, Brasil, E-mails: rafael_saraiva@ig.com.br, lisandro@uerj.br. alternativos podem atuar como fallback, quando métodos mais precisos - como o GPS (Global Positioning System) - estiverem indisponíveis devido a limitações sistêmicas - por exemplo, em ambientes indoor ou em áreas urbanas densas, onde a recepção de sinais GPS é muitas vezes inviável. A utilização dos métodos de localização de MS legados como fallback aumenta a disponibilidade dos serviços LCS. Esses métodos devem usar apenas informações já disponíveis na rede GSM ou fornecidas pelo MS através do relatório de medidas (MR measurement report).

$\mathrm{Na}$ hierarquia de rede GSM, o nível mais alto e de menor resolução para o rastreamento do MS corresponde à área geográfica atendida pela PLMN (Public Land Mobile Network) à qual o MS pertence. Associado à PLMN há um banco de dados denominado HLR (Home Location Register), que armazena as informações de todos os MS pertencentes àquela PLMN. A PLMN é dividida em uma ou mais MSC (Mobile Switching Center). Associado a cada MSC há um banco de dados denominado VLR (Visitor Location Register), que armazena as informações dos usuários registrados na MSC. Em cada MSC, uma ou mais BSC (Base Station Controller) controlam conjuntos de BTS (Base Transmission Station), que por sua vez comunicam-se diretamente com o MS. Diversas BTS podem ser agrupadas em uma LA (Location Area). O objetivo da LA é definir a região de procura por um MS quando há necessidade de terminação de uma chamada no mesmo. Por fim, cada BTS pode ser dividida em vários setores. Cada setor ou célula é identificado univocamente pelo CGI (Cell Global Identifier). A área geográfica coberta por cada setor depende de uma variedade de fatores, tais como ambiente de propagação, altura e tipo das antenas, potência de saída, etc. Essa estrutura hierárquica de localização de um MS é empregada tanto para permitir a terminação de chamadas em um MS (MS em modo idle) quanto para a continuidade de chamadas em curso (MS em modo ativo). Quando o MS está em modo ativo essas informações de localização são empregadas para controlar os parâmetros da interface aérea e para assessorar o processo de handoff (troca de setor servidor). Quando o MS está em modo idle, a melhor resolução para a localização é a área geográfica correspondente a LA. Para um MS em conversação (i.e., em modo ativo) um posicionamento muito mais preciso pode ser obtido. Durante o curso da chamada, informações sobre a rede servidora são coletadas pelo MS e reportadas através do MR. Neste trabalho explorase o uso do alinhamento temporal e dos parâmetros do MR, combinados com predições de cobertura, para localizar um MS em modo ativo.

Para a localização de terminais legados, as informações presentes no MR devem ser correlacionadas a informações armazenadas em um banco de dados. Esse banco de dados 
pode ser construído através de extensas medidas de campo (coletando milhares de MR) [5] ou através de predição de cobertura. Neste trabalho, optou-se pela segunda alternativa, uma vez que esta é uma opção muito mais barata e rápida para construir e atualizar o banco de dados. Em redes reais, mudanças ocorrem constantemente: re-azimute de antenas, modificação das potências de transmissão, ativação de novas BTS, etc. Bancos de dados construídos a partir de medidas não podem acomodar rapidamente essas alterações, tornandose obsoletos e requerendo novos dados de campo. Bancos de dados construídos a partir de modelagem de propagação podem ser rapidamente atualizados para reproduzir as alterações na rede. Uma possível desvantagem desta alternativa - uma relativa degradação de precisão devido a erros de predição de cobertura - pode ser minimizada através de adequada calibração dos modelos de propagação.

\section{PRedição de Cobertura}

\section{A. Modelo de Propagação}

O modelo de propagação utilizado para construir o mapa de predição de cobertura ou de nível de sinal recebido (RSS - Received Signal Strength) foi o de Okumura-Hata [6], largamente aplicado no planejamento de RF de redes celulares. Nele, a perda de propagação média é calculada via

$$
L=k_{1}+k_{2} \log (d)+k_{3} \log \left(h_{b}\right)+k_{4} L_{f}+k_{5} \log \left(h_{b}\right) \log (d),(1)
$$

onde $d$ é a distância em metros entre a BTS e o MS, $h_{b}$ é a altura efetiva da antena da BTS e $L_{f}$ é a atenuação por difração, calculada pelo método de Epstein-Peterson [7]. Assume-se que a altura do MS é igual a $1.5 \mathrm{~m}$. Os coeficientes $k_{i}$ dependem da morfologia e da freqüência de transmissão. Selecionamos a freqüência de $875 \mathrm{MHz}$, que é a frequência central da banda concedida à operadora móvel celular cuja rede foi utilizada no teste. Os valores empregados no modelo são $k_{1}=-12.1$ (urbano) e $k_{1}=-2.2$ (suburbano), $k_{2}=-44.9, k_{3}=-$ $5.83, k_{4}=0.5$ (urbano) e $k_{4}=0.4$ (suburbano), e $k_{5}=6.55$. A calibração inicial setor a setor desses coeficientes, a partir de medidas de campo, pode melhorar a precisão da atenuação predita pelo modelo. Medidas de campo também podem ser utilizadas para classificar a morfologia (urbana densa, urbana, suburbana, rural, etc) da área de melhor servidor de cada célula, auxiliando na seleção do conjunto de coeficientes mais adequado para cada setor. Um algoritmo de classificação morfológica a partir de parâmetros do MR é proposto em [8].

\section{B. Construção do mapa de predição cobertura}

Para que seja possível construir o mapa de predição de RSS, a topografia da região onde o serviço LCS será oferecido deve ser representada por meio de uma matriz $\mathcal{T}=\left(a_{i, j}\right)_{M \times N}$, onde $a_{i, j} \in \Re_{+}$para todo e qualquer $i \in\{1,2,3, \ldots, M\}$ e $j \in\{1,2,3, \ldots, N\}$. Cada elemento $a_{i, j}$ armazena a altitude média do terreno em um quadrado com $r$ metros de lado. Alternativamente, a matriz $\mathcal{T}$ pode conter, sobreposta à altitude do relevo, as alturas das edificações. Se a região cobre uma superfície total de $L \times A \mathrm{~m}^{2}$, então a matriz $\mathcal{T}$ terá $\left\lceil\frac{L}{r}\right\rceil \times$ $\left\lceil\frac{A}{r}\right\rceil$ elementos, os quais denominaremos pixels. Chamaremos o parâmetro $r$ de resolução da matriz $\mathcal{T}$.

A predição de RSS para uma antena isolada é obtida pela aplicação da Eq. (1) aos perfis do relevo entre a posição da antena e cada pixel na região de interesse. $\mathrm{Na}$ prática, para limitar a quantidade de perfis analisados, a região de cálculo pode ser restringida - por exemplo, podem ser calculadas as atenuações de propagação apenas para os pixels dentro de um círculo centrado na antena transmissora e com raio especificado. As características da antena transmissora - posição, azimute, diagrama de irradiação, potência isotrópica efetivamente irradiada (EIRP - Effective Isotropic Irradiated Power), etc - são consideradas na predição. $O$ mapa de predição $\mathcal{P}$ é obtido pela sobreposição das coberturas individuais das antenas na região de interesse. A matriz $\mathcal{P}$ tem resolução $r^{\prime}$, onde $r^{\prime} \geq r$. A Fig. 1 mostra a predição de RSS de uma antena isolada, utilizando uma matriz $\mathcal{P}$ com $r^{\prime}=10 \mathrm{~m}$ calculada sobre uma matriz $\mathcal{T}$ com $r=5 \mathrm{~m}$ e alturas das edificações sobrepostas ao relevo. Para melhor visualização, o RSS apresentado nessa figura está quantizado com passo maior do que aquele empregado na predição. A Fig. 1 mostra também os polígonos do prédios e os vetores das ruas na região.

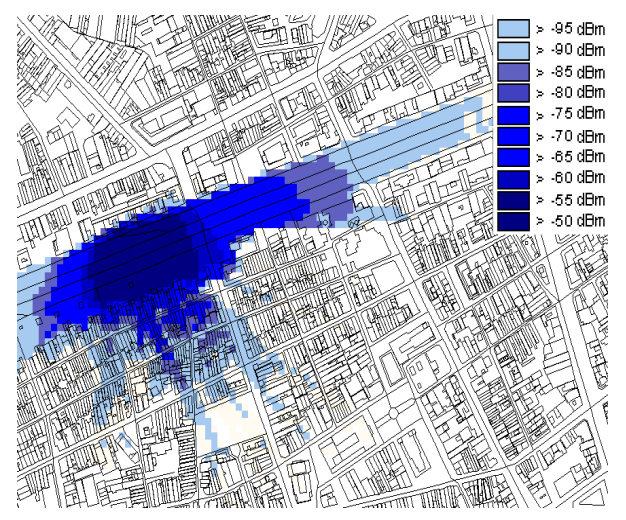

Fig. 1. Predição de RSS para uma antena isolada.

\section{LOCALIZAÇÃo DE TERMINAIS GSM LEGADOS}

A seguir são descritos os métodos avaliados neste trabalho. Estes métodos utilizam bancos de dados de RSS gerados a partir de predição de cobertura devido à maior praticidade dessa abordagem relativamente à construção desses bancos a partir de medições.

\section{A. Métodos usando identidade da célula e alinhamento tem- poral}

O primeiro parâmetro do MR que pode ser utilizado para estimar a localização do MS é a identidade da célula servidora (CI - Cell Identifier). O CI é parte do CGI. Como uma primeira aproximação, pode-se assumir que o MS está localizado nas coordenadas da antena servidora [9], [10]. Para reduzir o erro de posicionamento, pode-se utilizar o centróide da área geográfica coberta pela célula servidora como estimativa da posição do MS. Para uma célula setorizada, essa área pode ser representada por uma seção angular centrada na antena servidora e definida pelo azimute e diagrama de radiação horizontal da antena servidora [9], [10]. Porém, tal representação é uma simplificação. Na prática, a área de melhor servidor de cada setor é irregular e depende do ambiente de propagação e também das características dos sistemas irradiantes da célula 
servidora e das células vizinhas. Uma representação mais realista pode ser obtida através de mapas de cobertura construídos com modelos de propagação empíricos e bases de dados topográficas digitalizadas. O método que utiliza o centróide da área de melhor servidor - obtida através de predição de cobertura - como estimativa de posição do MS é denominado CI-PRED [11].

O alinhamento temporal dinâmico é utilizado em redes GSM para evitar a colisão - na antena receptora do setor servidor - de rajadas de dados transmitidas por diferentes MS. Para isso, utiliza-se o parâmetro TA (Timing Advance), que é expresso em múltiplos inteiros do período de bit GSM, assumindo valores de 0 a 63. O parâmetro TA pode ser relacionado à distância percorrida pelo sinal entre a antena servidora e o MS via [12]

$$
\mathrm{TA}=\operatorname{int}\left(\frac{2 d / c}{\mathrm{~T}_{b}}\right)
$$

onde $c$ é a velocidade da luz no vácuo, $d$ é o comprimento do percurso e $\mathrm{T}_{b}$ é o período de bit GSM (3.69 $\left.\mu \mathrm{s}\right)$. Então, a partir do valor de TA pode-se calcular uma estimativa de distância de visada direta (comprimento do percurso do sinal) entre o MS e a antena servidora dada por

$$
\hat{d}=\frac{c \mathrm{~T}_{b} \mathrm{TA}}{2} \text { metros. }
$$

O parâmetro TA pode ser utilizado para melhorar a estimativa de localização do método CI-PRED. Para tal, as áreas de melhor servidor de cada setor - obtidas a partir do mapa de predição de cobertura - são subdividas conforme valores de TA preditos. $\mathrm{O}$ valor de TA em cada pixel do mapa de cobertura é predito pela Eq. (2), inserindo na variável $d$ a distância de visada direta entre o pixel e a antena servidora. Para cada dupla $\left(C I_{i}, T A_{P, j}\right)$ haverá um par de coordenadas $\left(x_{i, j}, y_{i, j}\right)$, correspondente ao centróide da $j$-ésima subárea de TA predito pertencente à área de melhor servidor da $i$ ésima célula. Este par de coordenadas será a estimativa de posição produzida para uma dupla $(C I, T A)$ medida pelo MS, onde $(C I, T A)=\left(C I_{i}, T A_{P, j}\right)$. Este método de estimativa da localização de um MS é denominado CI+TA-PRED [11].

$\mathrm{O}$ valor predito de TA pode divergir do valor real, especialmente em condições de propagação sem visada direta (NLOS - Non Line of Sight Propagation). Isso ocorre porque $d$ na Eq. (2) representa o percurso do sinal entre o MS e a antena servidora e não a distância direta entre ambos, que é utilizada para predizer o valor de TA associado a cada pixel do mapa de predição.

\section{B. Métodos usando correlação de assinaturas de RF}

A assinatura de RF medida é composta por um subconjunto dos parâmetros disponíveis no $\mathrm{MR}$, acrescido do valor de TA. Este subconjunto contém o BCCH (Broadcast Control Channel), o BSIC (Base Station Identity Code) e o RXLEV (Received Signal Level) do melhor servidor e de até 6 setores vizinhos cujos sinais são recebidos com maior intensidade. Para cada vizinho listado no MR, o vetor coluna $[\mathrm{BCCH} \mathrm{BSIC}]^{\mathrm{T}}$ associado é convertido no valor de CI correspondente para identificação unívoca da célula GSM.

Seja $\mathcal{S}$ o conjunto de todos os elementos da matriz de RSS preditos $\mathcal{P}$. Para cada pixel, a assinatura de RF predita é calculada através de modelagem de propagação, isto é, utilizando $\mathcal{P}$. Assim, a assinatura do $i$-ésimo elemento é o par $\left(T A_{P, i}, \dot{\hat{\nu}}_{P, i}\right)$, onde $T A_{P, i}$ é a estimativa do valor de TA (calculada como no método CI+TA-PRED) e $\hat{\nu}_{P, i}$ é a matriz

$$
\hat{\nu}_{P, i}=\left[\begin{array}{cc}
\mathrm{CI}_{P, 1} & \operatorname{RXLEV}_{P, 1} \\
\vdots & \vdots \\
\mathrm{CI}_{P, N} & \operatorname{RXLEV}_{P, N}
\end{array}\right] .
$$

A matriz $\hat{\nu}_{P, i}$ tem dimensões $N \times 2$, onde $N$ varia de 1 (quando há apenas um setor servidor) a 7 (melhor servidor mais seis setores vizinhos). Cada linha contém o CI e o RXLEV correspondente, obtido através de modelagem de propagação. O banco de dados de assinaturas de RF preditas está completo quando os $\left(T A_{P, i}, \hat{\nu}_{P, i}\right)$ de todos os elementos da matriz $\mathcal{P}$ foram calculados.

Para estimar a posição do MS deve-se proceder à correlação entre as assinaturas de RF medida e predita. Pode-se assumir que o MS está localizado na posição do pixel cuja assinatura de RF predita mais se assemelha à assinatura de RF medida. Entretanto, esta abordagem pode induzir a desvios devido aos erros de predição e à imprecisão das medidas do MS [11]. Então, ao invés de considerar apenas um pixel na estimativa de posição do MS, pode-se utilizar a média aritmética das coordenadas de um conjunto de pelo menos $K$ pixels, aqueles com as assinaturas de RF preditas mais semelhantes à assinatura de RF reportada pelo MS. Para avaliar a similaridade entre as assinaturas de RF medida e predita pode-se calcular o quadrado da distância Euclidiana entre as duas. Entretanto, não seria factível calcular tal distância para todos os pixels na área de serviço. Assim, algum processo de filtragem - que não piore o erro da estimativa de posição - é recomendável para reduzir a carga computacional. Neste trabalho propõem-se um processo de correlação de assinatura de RF que é composto de 4 passos sucessivos de filtragem:

1) Seleção dos pixels de $\mathcal{S}$ pertencentes à área de melhor servidor da célula identificada por $\mathrm{CI}_{M, 1}$, ou seja, obtém-se o conjunto de pixels $\mathcal{A}=$ $\left\{i \in \mathcal{S} \mid \mathrm{CI}_{P, i, 1}=\mathrm{CI}_{M, 1}\right\}$, onde $\mathrm{CI}_{P, i, 1}$ é o CI do melhor servidor predito na posição do $i$-ésimo pixel e $\mathrm{CI}_{M, 1}$ é o CI do melhor servidor medido e reportado no MR. Repare que $\mathcal{A} \subset \mathcal{S}$.

2) Seleção dos elementos de $\mathcal{A}$ com valor de TA predito igual ao valor de TA medido, ou seja, $\mathcal{B}=$ $\left\{i \in \mathcal{A} \mid \mathrm{TA}_{P, i}=\mathrm{TA}_{M}\right\}$, onde $\mathrm{TA}_{P, i}$ é o TA predito na posição do $i$-ésimo pixel e $\mathrm{TA}_{M}$ é o TA medido pela célula servidora. Repare que $\mathcal{B} \subset \mathcal{A} \subset \mathcal{S}$.

3) i) Definição do conjunto $\mathcal{F}_{i}$ : o conjunto $\mathcal{F}_{i}$ contém os CIs preditos na posição do $i$-ésimo pixel que são iguais aos CIs (melhor servidor e vizinhos) reportados no MR. Assim, se $\mathcal{I}_{P, i}$ é o conjunto de CIs preditos em $i$ e $\mathcal{I}_{M, i}$ é o conjunto de CIs medidos e reportados no $\mathrm{MR}$, então $\mathcal{F}_{i}=\mathcal{I}_{P, i} \cap \mathcal{I}_{M, i}$; ii) Seleção dos elementos de $\mathcal{B}$ que possuem ao menos $N$ valores de $\mathrm{CI}$ preditos iguais aos reportados no $\mathrm{MR}$, ou seja, $\mathcal{C}=$ $\left\{i \in \mathcal{B}, N \in[1,7] \mid N \leq \#\left(\mathcal{F}_{i}\right) \leq 7\right\}$, onde $\#\left(\mathcal{F}_{i}\right)$ é a cardinalidade de $\mathcal{F}_{i}$. Se nenhum pixel satisfizer essa condição, o valor de $N$ é decrementado até que $\mathcal{C}$ contenha ao menos 1 elemento. Repare que $\mathcal{C} \subset \mathcal{B} \subset$ $\mathcal{A} \subset \mathcal{S}$. 
4) Para cada pixel $i \in \mathcal{C}$, o quadrado da distância Euclidiana entre os valores de RXLEV medido $\left(\operatorname{RXLEV}_{C I_{M, k}}\right)$ e predito $\left(\mathrm{RXLEV}_{C I_{P, i, k}}\right)$

$$
d_{i}=\sum_{C I \in \mathcal{F}_{i}}\left[\operatorname{int}\left(\left(\operatorname{RXLEV}_{C I_{M}}-\operatorname{RXLEV}_{C I_{P, i}}\right) \delta^{-1}\right)\right]^{2}
$$

é calculada para cada $\mathrm{CI}_{k} \in \mathcal{F}_{i}\left(k \in\left[1, \#\left(\mathcal{F}_{i}\right)\right]\right)$. Na Eq. (5), $\delta$ representa a imprecisão na medida de RXLEV pelo MS [12] ( $\pm 6 \mathrm{~dB})$. Apenas os pixels com as $K$ menores diferenças são selecionados.

A estimativa de posição do MS é dada pela média aritmética das coordenadas dos pixels restantes após o $4^{\circ}$ passo de filtragem. Denominaremos este método de localização RF-FING+TA-PRED. Esta abordagem é um refinamento do método RF-FING-PRED [11]. A utilização do parâmetro TA deverá reduzir a incerteza no posicionamento bem como acelerar o processo de filtragem (ver Seção IV-B.4). Cabe ressaltar que dois aspectos do método RF-FING+TA-PRED e de relevância prática na implantação de serviços LCS não foram encontrados na literatura: i) a consideração do erro de medida do MS e ii) a filtragem de pixels utilizando subáreas - definidas sobre a área de melhor servidor predita - com TA predito constante. Uma forma alternativa de utilização do TA para a filtragem é proposta em [13], onde o TA é usado para limitar a distância máxima entre os pixels (a serem utilizados no processo de correlação) e a antena transmissora.

\section{RESUltados EXPERIMENTAIS}

\section{A. Configuração do teste}

Testes foram realizados nos bairros Centro (Área 1) e Santa Cruz (Área 2) do município do Rio de Janeiro para avaliar as melhorias decorrentes das modificações propostas frente aos métodos apresentados em [11]. Um resumo das características dessas áreas pode ser visto na Tab. I. O conjunto de equipamentos de teste incluía um telefone GSM legado e um receptor GPS, ambos conectados a um laptop instalado no interior de um veículo. O MS estava em modo ativo e para cada MR transmitido o valor de TA foi identificado e a posição do MS foi calculada via GPS. Foram coletados 4501 MRs na Área 1 e 7864 MRs na Área 2. Essa quantidade de amostras provê confiabilidade razoável às estatísticas obtidas. A localização calculada pelo GPS foi tomada como referência, então, para cada MR, o erro de posicionamento é a distância Euclidiana entre a posição fornecida pelo GPS e a posição estimada por cada método.

TABELA I

CARACTERÍSTICAS DAS ÁREAS DE TESTE.

\begin{tabular}{|l||c|c|}
\hline Característica & Área 1 & Área 2 \\
\hline \hline Morfologia & Urbana & Suburbana \\
\hline Área & $4.8 \mathrm{~km}^{2}$ & $305 \mathrm{~km}^{2}$ \\
\hline $\begin{array}{l}\text { Densid.sup. } \\
\text { de setores }\end{array}$ & $23.75 \mathrm{set} / \mathrm{km}^{2}$ & $0.07 \mathrm{set} / \mathrm{km}^{2}$ \\
\hline $\begin{array}{l}\text { Resolução da base } \\
\text { topográfica ( } \mathrm{r})\end{array}$ & $5 \mathrm{~m}$ & $25 \mathrm{~m}$ \\
\hline $\begin{array}{l}\text { Altimetria } \\
\text { de prédios? }\end{array}$ & Sim & Não \\
\hline
\end{tabular}

\section{B. Análise dos Resultados}

$\mathrm{Na}$ seção III foram discutidos quatro métodos de localização: CI-PRED, CI+TA-PRED, RF-FING-PRED e RFFING+TA-PRED; sendo o quarto uma contribuição deste trabalho. Os dois últimos utilizam correlação de assinatura de RF e possuem desempenhos que variam conforme: i) a quantidade de servidores $N$ considerada na procura de assinaturas semelhantes, e ii) a quantidade de assinaturas $K$ utlizada para o cálculo da estimativa de posição . Os resultados nesta subseção foram obtidos com o melhor par $(N, K)$, ou seja, aquele que produz o menor erro de localização para cada método em cada área de teste. Na subseção IV-B.2 é apresentado um estudo da influência desses valores nos algoritmos de localização baseados em correlação de assinatura de RF.

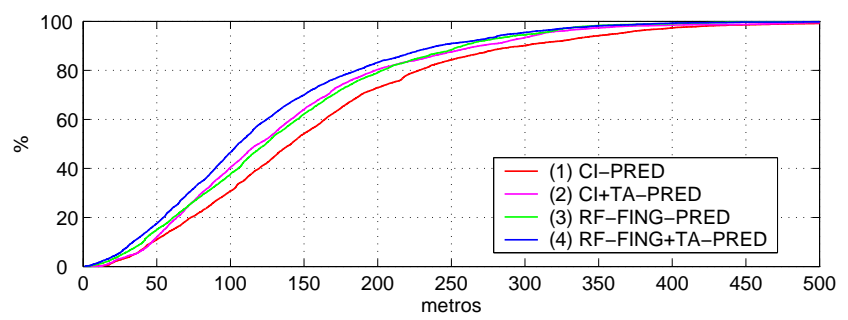

Fig. 2. CDF do erro de localização (em metros) na Área 1.

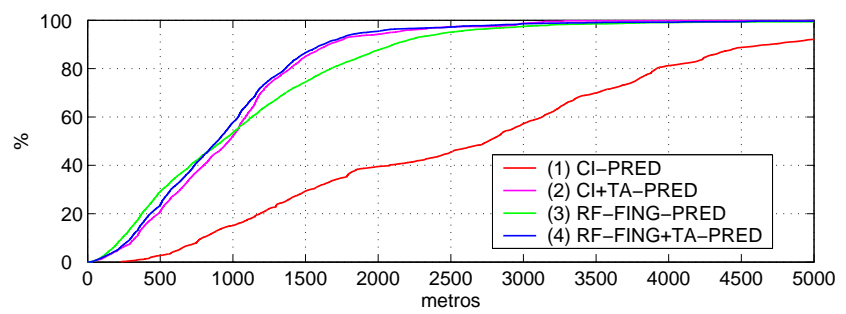

Fig. 3. CDF do erro de localização (em metros) na Área 2.

A Fig. 2 mostra a função de distribuição de probabilidade cumulativa (CDF - Cumulative Distribution Function) do erro de localização na Área 1 para $r^{\prime}=10 \mathrm{~m}$. O método RFFING+TA-PRED $(N=1, K=2)$ produziu os melhores resultados, com erro médio de 125 metros e desvios de 105, 141 e 294 metros para o $50^{\circ}, 67^{\circ}$ e $95^{\circ}$ percentis, respectivamente. A utilização do método RF-FING-PRED $(N=2, K=4)$ acarreta um erro médio $10 \%$ maior.

A Fig. 3 mostra a CDF do erro de localização na Área 2 para $r^{\prime}=200 \mathrm{~m}$. O método RF-FING+TA-PRED $(N=2, K=2)$ obteve o melhor desempenho, com erro médio de 970 metros e desvios de 894,1124 e 1924 metros para o $50^{\circ}, 67^{\circ}$ e $95^{\circ}$ percentis, respectivamente. Os erros maiores na Área $2 \mathrm{em}$ comparação à Área 1 devem-se à baixa densidade de setores na região, conforme pode ser observado na Tab. I. Esta também é a razão pela qual TA $>2$ em mais de $40 \%$ dos registros coletados na Área 2, enquanto na Área 1 cerca de $98 \%$ continham TA $=0$. Por isso, a inserção do TA nos métodos RF-FING+TA-PRED $(N=2, K=2)$ e CI-PRED acarreta um ganho de acurácia (redução do erro de localização) relativamente maior na Área 2 do que na Área 1. 
1) Comparação com Resultados Anteriores: Erros de 94 e 291 metros para o $50^{\circ}$ e $90^{\circ}$ percentis foram relatados em [5] para um método de correlação utilizando banco de dados de assinaturas de RF construído a partir de medidas de campo em uma área urbana densa com 66 setores $/ \mathrm{km}^{2}$. $\mathrm{Na}$ Área 1, com 23 setores $/ \mathrm{km}^{2}$, o método RF-FING+TA$\operatorname{PRED}(N=1, K=2)$ produziu erros de 105 e 240 metros para os mesmos quantis. Para comparar a precisão dos dois métodos, o erro de RF-FING+TA-PRED $(N=1, K=2)$ foi normalizado em função da maior densidade de setores, conforme procedimento proposto em [11]. Após a normalização, o erro equivalente de RF-FING+TA-PRED $(N=1, K=2)$ foi de 80 e 182 metros para o $50^{\circ}$ e $95^{\circ}$ percentis, ficando abaixo daqueles obtidos em [5].

2) Valores ótimos para $N$ e $K$ : Em um cenário hipotético, onde fosse possível modelar com exatidão o ambiente de rádio-propagação e onde o RSS médio em uma dada posição fosse invariante no tempo, a dupla $(N, K)$ que maximizaria a precisão seria $(7,1)$. Ou seja, haveria equivalência entre todos os CIs medidos e preditos e seria selecionado apenas o pixel com a menor diferença quadrática (que seria nula) como estimativa da posição do MS. Porém, em um cenário real, a confiabilidade da predição de RSS é maior para os sinais mais fortes. Então, para $N$ grande, além de apenas poucos pontos serem selecionados no $3^{\circ}$ passo de filtragem, os pixels escolhidos terão uma matriz $\hat{\nu}_{P}$ menos confiável, uma vez que vizinhos mais fracos serão incluídos. Assim, espera-se que um valor de $N$ menor forneça os melhores resultados. De fato, a máxima precisão foi obtida para $N=1$ (apenas o melhor servidor) na Área 1 e $N=2$ (melhor servidor mais 1 vizinho) na Área 2, independentemente da resolução $r^{\prime}$ do mapa de predição. O maior valor de $N$ ótimo na Área 2 reflete a melhor representação do ambiente de propagação na zona suburbana, pois há menos reflexões e predominância de propagação em visada direta (LOS - Line of Sight). Verificou-se que o valor ótimo de $K$ para RF-FING+TA-PRED varia inversamente com a resolução $r^{\prime}$ na Área 1 , indo de $K=3$ para $r^{\prime}=5 \mathrm{~m}$ até $K=1$ para a $r^{\prime}=25 \mathrm{~m}$. Suspeita-se que isso ocorre pois um pixel maior elimina certas variações espaciais (dentro da área $r^{\prime} \times r^{\prime} \mathrm{m}^{2}$ do pixel) do valor predito de RSS. Ou seja, para $r^{\prime}$ maior, um melhor resultado é obtido sem a necessidade de considerar a média aritmética das coordenadas de vários pixels - o que seria o caso para $r^{\prime}$ menor, onde $K$ ótimo é maior que 1 . Na Área 2, o valor ótimo de $K$ é igual a 2 para todas as resoluções testadas para a construção do mapa de predição. Os resultados sugerem que os valores de $N$ e $K$ ótimos estão relacionados à qualidade da predição de RSS, e que o $K$ ótimo é função também da resolução do mapa de predição de cobertura. A Fig. 4 mostra o erro de localização médio e o erro do $67^{\circ}$ percentil para valores de $(N, K) \in[1,6] \times[1,5]$ para o método RF-FING+TAPRED, com $r^{\prime}=10 \mathrm{~m}$ na Área $1 \mathrm{e} r^{\prime}=200 \mathrm{~m}$ na Área 2. Os pares que minimizam simultaneamente esses erros de posicionamento nas resoluções indicadas são $(1,2)$ na Área 1 e $(2,2)$ na Área 2.

3) Influência de $r^{\prime}$ na Acurácia de Localização: Os métodos apresentados nas seções III-A e III-B utilizam modelagem de propagação. Logo, sua acurácia é afetada pela resolução $r^{\prime}$ do mapa de predição de RSS. Para otimizar o
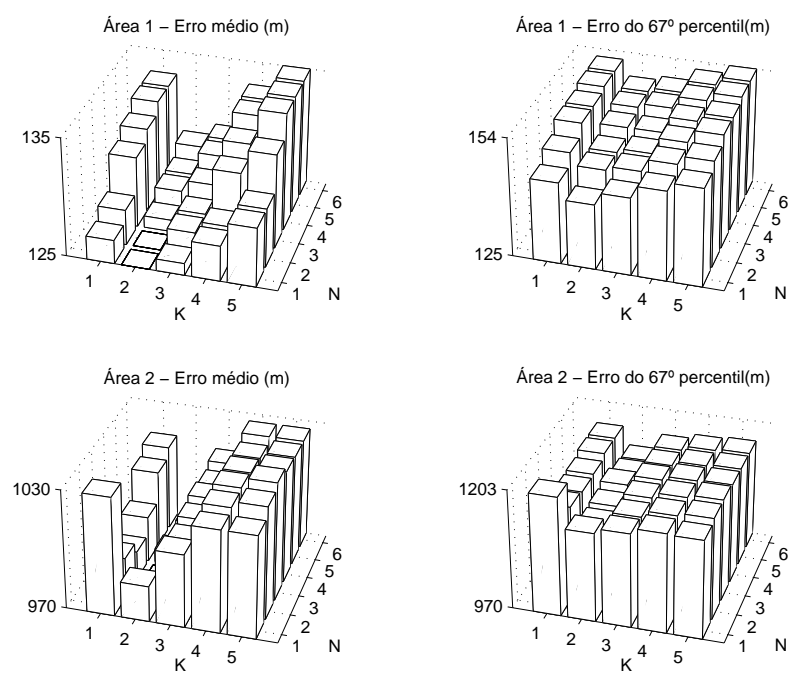

Fig. 4. Erro de localização do método RF-FING+TA-PRED para diferentes valores de $(N, K)$.

desempenho sem perder a qualidade, deve-se aplicar o maior valor de $r^{\prime}$ para o qual não ocorre degradação da acurácia da localização do MS. Em função deste valor máximo para a resolução do mapa de RSS $\left(r_{\text {max }}^{\prime}\right.$ ) define-se o fator de redução de custo computacional, que é dado pela razão $\left(r_{\max }^{\prime} / r_{\min }^{\prime}\right)^{2}$, onde $r_{\text {min }}^{\prime}=r$.

A Tab. II mostra o erro de localização para o $50^{\circ}, 67^{\circ}$ e $95^{\circ}$ percentis nas duas áreas de teste, para diferentes valores de $r^{\prime}$. Na Área 2, independentemente do método, a degradação da acurácia em função de $r^{\prime}$ é desprezível para todas as resoluções testadas. Na Área 1, observa-se o mesmo comportamento para os métodos CI-PRED e CI+TA-PRED. Para os métodos RF-FING-PRED $(N=2, K=4)$ e RFFING+TA-PRED $(N=1, K=2)$ (na Área 1 , o valor de $r^{\prime}$ não pode ser aumentando acima de 10 metros sem comprometer a acurácia da localização. Assim, o fator de redução de custo computacional é igual a $64\left(r_{\max }^{\prime}=200 \mathrm{~m}, r=25 \mathrm{~m}\right)$ na Área 2 e igual a $4\left(r_{\max }^{\prime}=10 \mathrm{~m}, r=5 \mathrm{~m}\right)$ na Área 1 . Este menor fator de redução na Área 1 deve-se à presença da altimetria de prédios na base topográfica digitalizada representando esta região.

TABELA II

ERRO DE LOCALIZAÇÃo VERSUS $r^{\prime}$ PARA OS MÉTODOS 1)CID-PRED , 2)CID+TA-PRED, 3)RF-FING-PRED E 4)RF-FING+TA-PRED.

\begin{tabular}{|c|c|c|c|c|c|c|c|c|c|}
\hline \multicolumn{10}{|c|}{ Área 1 (erro em metros) } \\
\hline$r^{\prime}$ & \multicolumn{3}{|c|}{$5 \mathrm{~m}$} & \multicolumn{3}{|c|}{$10 \mathrm{~m}$} & \multicolumn{3}{|c|}{$25 \mathrm{~m}$} \\
\hline $\mathrm{M}$ & $50 \%$ & $67 \%$ & $95 \%$ & $50 \%$ & $67 \%$ & $95 \div$ & $50 \div$ & $67 \%$ & $95 \%$ \\
\hline 1 & 142 & 183 & 362 & 141 & 181 & 363 & 140 & 186 & 360 \\
\hline 2 & 119 & 157 & 311 & 119 & 157 & 312 & 121 & 159 & 318 \\
\hline 3 & 118 & 157 & 317 & 124 & 162 & 307 & 128 & 169 & 327 \\
\hline 4 & 105 & 139 & 292 & 105 & 141 & 294 & 115 & 149 & 304 \\
\hline \multicolumn{10}{|c|}{ Área 2 (erro em km) } \\
\hline$r^{\prime}$ & \multicolumn{3}{|c|}{$25 \mathrm{~m}$} & \multicolumn{3}{|c|}{$100 \mathrm{~m}$} & \multicolumn{3}{|c|}{$200 \mathrm{~m}$} \\
\hline $\mathrm{M}$ & $50 \%$ & $67 \%$ & $95 \%$ & $50 \%$ & $67 \%$ & $95 \%$ & $50 \div$ & $67 \%$ & $95 \%$ \\
\hline 1 & 2.8 & 3.3 & 5.5 & 2.8 & 3.4 & 5.6 & 2.8 & 3.4 & 5.5 \\
\hline 2 & 1.0 & 1.2 & 2.1 & 1.0 & 1.2 & 2.2 & 1.0 & 1.2 & 2.1 \\
\hline 3 & 1.0 & 1.3 & 2.5 & 1.0 & 1.3 & 2.5 & 1.0 & 1.3 & 2.5 \\
\hline 4 & 0.9 & 1.2 & 1.9 & 1.0 & 1.2 & 2.0 & 0.9 & 1.2 & 2.0 \\
\hline
\end{tabular}

4) Redução do tempo de processamento: Para um mesmo conjunto de valores de $r^{\prime}, N$ e $K$, o método RF-FING+TA- 
PRED reduz o tempo de processamento relativamente ao método RF-FING-PRED em cerca de 30\% na Área 1 e $90 \%$ na Área 2. Este cálculo foi feito considerando o tempo necessário para estimar a posição de todos os MRs coletados em cada área. A comparação foi feita utilizando o valor $(N, K)$ ótimo e $r^{\prime}=r_{\max }^{\prime}$ em cada área. Estes resultados mostram que, quanto menor a densidade de setores em uma região - e, consequentemente, maior o número médio de pixels por área de melhor servidor - maior a redução percentual do tempo de processamento.

5) Influência da qualidade de predição na acurácia de localização: Seja um ponto localizado nas coordenadas $\left(\mathrm{x}_{i}, \mathrm{y}_{j}\right)$. Nesse ponto, seja $\mathrm{CI}_{M}$ o setor melhor servidor medido pelo MS e $\mathrm{TA}_{M}$ o valor de TA atribuído ao MS pelo setor servidor. Pode-se afirmar que: i) se a predição de cobertura for suficientemente precisa, o setor melhor servidor predito $\left(\mathrm{CI}_{P}\right)$ será igual a $\mathrm{CI}_{M}$; ii) se condições de propagação LOS forem predominantes e não houver erro devido à quantização do valor de TA, então o valor de TA predito pela Eq. (2) $\left(\mathrm{TA}_{P}\right)$ será igual a $\mathrm{TA}_{M}$. Se estas duas condições ocorrerem em um dado ponto, espera-se que a acurácia da localização baseada em predição de cobertura e alinhamento temporal seja melhor neste ponto. Do total de MRs coletados na Área 1, em aproximadamente $44 \%$ deles a primeira condição é verdadeira e em $40 \%$ ambas ocorrem. O erro médio de localização dos métodos CID+TA-PRED e RF-FING+TA-PRED $(N=1, K=2)$ para o subconjunto de pontos de medida onde ambas são verdadeiras é de 78 e 74 metros, respectivamente. Na Área 2, a primeira condição ocorre em $78 \%$ dos pontos de medida. Este percentual maior deve-se à melhor modelagem do ambiente de propagação na zona suburbana. Nessa área as duas condições verificam-se em $66 \%$ dos pontos de medida. Para estes, a diminuição observada no erro médio dos métodos CID+TAPRED e RF-FING+TA-PRED $(N=2, K=2)$ foi de $4 \%$ e $8 \%$, respectivamente, bem menores do que as obtidas na Área 1. Isso é reflexo da baixa densidade de setores na Área 2. Estes resultados sugerem que a precisão dos métodos CID+TA-PRED e RF-FING+TA-PRED pode ser melhorada se os modelos de propagação utilizados forem calibrados, especialmente em áreas com maior densidade de setores.

6) Influência do valor de TA na acurácia média da localização: $\mathrm{Na}$ Área 1, os erros médios de localização obtidos pelo método CI+TA-PRED nos pontos de teste com $\mathrm{TA}=0$ e $\mathrm{TA}=1$ são 137 e 268 metros, respectivamente. Para o RF-FING+TA-PRED $(N=1, K=2)$ eles caem para 124 e 164 metros. A Fig. 5 mostra o erro médio de localização em função do TA medido na Área 2. Esses dados indicam que a acurácia média da estimativa de localização diminui com o aumento do TA em ambas as áreas, o que está de acordo com [10]. Tal comportamento pode ser explicado através da representação geométrica simplificada descrita em [9] e [10], onde a região na qual o MS pode estar localizado é definida como uma seção angular com orientação e abertura definidas pelas características da antena transmissora e largura igual à resolução espacial de TA $\left(c T_{b} / 2 \approx 550 \mathrm{~m}\right)$. À medida que o valor de TA aumenta, a superfície desta região cresce e com ela a incerteza da estimativa de posição.

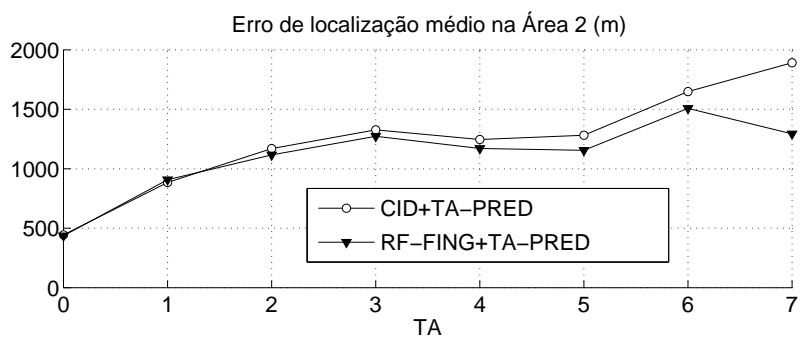

Fig. 5. Erro de localização médio na Área 2 em função do valor de TA.

\section{Conclusões}

Neste trabalho foram analisados métodos de localização de terminais móveis GSM legados. Esses métodos empregam informações já presentes na rede GSM para localizar os terminais sem requerer características adicionais dos mesmos. As estimativas de posição são obtidas através da correlação de parâmetros disponíveis no relatório de medidas enviado pelo móvel com assinaturas de rádio-freqüência. Investigou-se a utilização de modelagem de rádio-propagação para construir os bancos de dados de assinaturas de rádio-frequiência, ao invés de exaustivas medições. Essa abordagem é justificada pelo menor custo, maior facilidade e maior capacidade de acomodação de mudanças usando modelagem de propagação na construção desses bancos, relativamente a bancos construídos a partir de medidas de campo. Testes de campo avaliaram o desempenho dos diferentes métodos em cenários reais. Propôs-se uma alternativa para a utilização do alinhamento temporal do terminal móvel com a célula servidora conjuntamente à informações de níveis de potência dos sinais recebidos. Essa alternativa permite reduzir tanto a complexidade do algoritmo de estimação da posição do terminal quanto o erro de localização.

\section{REFERÊNCIAS}

[1] FCC, FCC Wireless Enhanced E911 Requirements. USA, 1999.

[2] European Commission, "Commission Recommendation on the processing of caller location information in electronic communication networks for the purpose of location-enhanced emergency call services 2003/558/EC", Official Journal of the European Union, Julho 2003.

[3] ETSI TS 101724, v8.9.0 (2004-06), Digital telecommunications system (Phase 2+); Location Services (LCS); Functional description; Stage 2 (3GPP TS 03.71 version 8.9.0 Release 1999). ETSI, 2004.

[4] TELECO, http://www.teleco.com.br/ncel.aps. Acesso em 7 abr. 2008, 19:40:00.

[5] M. Chen and T. Sohn et. al., Practical Metropolitan-scale Positioning for GSM Phones. Ubicomp, 2006.

[6] M. Hata, "Empirical Formula for Propagation Loss in Land Mobile Radio Services", IEEE Transactions on Vehicular Technology, vol. 29, no. 3, Sep. 1980.

[7] M. D. Yacoub, Foundations of Mobile Radio Engineering. CRC Press, 1993.

[8] O. Turkyilmaz, F. Alagoz, G. Gur and T. Tugcu, "Environment Aware Location Estimation in Cellular Networks", EURASIP Journal on advances in Signal Processing, vol. 2008, Article ID 276456, 9 pages, 2008.

[9] M. Weckström and M. Spirito and V. Ruutuu, "Mobile Station Location", GSM, GPRS and EDGE Performance. John Wiley and Sons, 2002, pp. $119-141$.

[10] M. A. Spirito and S. Pöykko and O. Knuuttila, "Experimental Performance of Methods to Estimate the Location of legacy Handsets in GSM", Proc. of IEEE Vehicular Tech. Conf., 2001.

[11] R. S. Campos e L. Lovisolo, "Location Methods for Legacy GSM Handsets using Coverage Prediction", 9th IEEE Workshop on Signal Processing Advances in Wireless Communications. Recife, Brazil, 2008.

[12] ETSI TS 100911 V6.2.0 (1998-07), Digital cellular telecommunications system (Phase 2+); Radio subsystem link control (GSM 05.08 version 6.2.0 Release 1997). ETSI, 1998.

[13] M. Khalaf-Allah e K. Kyamakya, "Mobile Location using Database Correlation with Least-Squares and Bayes Filtering", 12th European Wireless Conference. Athens, Greece, 2006. 